Urdinola, Z. (2017). Representaciones territoriales y heterotopías en Zarzal (Valle del Cauca-Colombia). Revista de Sociología y Antropología: VIRAJES, 19 (1), 125-146. DOI: 10.17151/rasv.2017.19.1.7

\title{
REPRESENTACIONES TERRITORIALES Y HETEROTOPÍAS EN ZARZAL (VALLE DEL CAUCA-COLOMBIA)*
}

\author{
ZAYRA URDINOLA HINCAPIÉ**
}

Recibido: 24 de diciembre de 2016 Aprobado: 12 de febrero de 2017

Artículo de investigación

\footnotetext{
* Producto del proceso de la tesis doctoral "Representaciones territoriales de la identidad de lugar a partir de procesos socioeconómicos en el valle interandino del río Cauca (Colombia)", directora: Dra. Beatriz Nates Cruz, Doctorado en Estudios Territoriales, Universidad de Caldas (Colombia)

** Magíster en Desarrollo Regional y Planificación de Territorios, coordinadora de Administración de Empresas Universidad del Valle sede Cartago y Candidata a Doctora. Zayra.2511112521@ucaldas.edu. co, Zayra.urdinola@correounivalle.edu.co, dirección postal: 762021- 762021056.
}

(1) ORCID: 0000-0003-3731-4559 


\title{
Resumen:
}

El objetivo de la investigación fue caracterizar las heterotopías en el municipio de Zarzal ubicado en el norte del Valle del Cauca (Colombia) a partir de las representaciones territoriales sobre las prácticas productivas y la territorialización del poder de los actores empresariales, con el fin de identificar los impactos en la evolución económica y la morfología del territorio. El método empleado fue inductivo con enfoque cualitativo de tipo descriptivo en la primera fase y en la segunda explicativo. Se realizó análisis del discurso en nueve entrevistas aplicadas a los actores sociales, etnografía -años 2011 a 2014- analizando prácticas en 20 actores empresariales y apoyados en fuentes documentales para el análisis diacrónico -período 1990 a 2010-, construyendo cartografía y sintaxis espacial. El resultado fue la caracterización de las heterotopías cuyas morfologías en el espacio social generan vínculos en la región. En conclusión, se evidencian tensiones socioespaciales en la representación de la relación territorio-apropiación de los actores empresariales y sociales en los intercambios de bienes/servicios inmersos en una lógica transaccional local de demanda funcional-global de procesos productivos industriales tecnificados sobre otros carentes de especialización.

Palabras clave: territorio, heterotopía, representaciones, actores empresariales, prácticas.

\section{TERRITORIAL REPRESENTATIONS AND HETEROTOPIAS IN ZARZAL, VALLE DEL CAUCA, COLOMBIA}

\begin{abstract}
:
The main objective of the research was to characterize the heterotopias in the municipality of Zarzal, located in the northern area of Valle del Cauca (Colombia) starting with territorial representations on the productive practices and the territorialization of the power of business actors, with the purpose of identifying the impact on economic development and morphology of the territory. The method used was inductive with qualitative approach of descriptive type in the first phase and explicative type in the second phase. A discourse analysis was conducted in nine interviews applied to social actors, ethnography -from 2011 to 2014- analyzing practices of twenty business actors and supported by documentary sources for diachronic analysis -from 1990 to 2010-, building cartography and spatial syntax. The result was the characterization of heterotopias whose morphologies in the social space generate links in the region. In conclusion, socio-spatial tensions are evidenced in the relationship territory-ownership of the social and business actors in the exchange of goods/services immersed in a local transactional logic of functional-global demand for more technical industrial production processes over other processes in need of specialization.
\end{abstract}

Key Words: territory, heterotopias, representations, corporate actors, practices. 


\section{Introducción}

— n el estudio nos ocupamos por objetivar la representación -1 territorial del vínculo que une a los actores con el lugar ${ }^{1}$ leído desde el proceso de significación social que corresponde a lo que (North, 1991, pp. 15, 17 y 34) llama estructura de poder y como ello hace que se destaque Zarzal en el contexto regional y nacional, caracterizando los procesos socioeconómicos en la década 1990 a 2010 a través del análisis relacional espacio-tiempo, allí encontramos que las prácticas industriales tecnificadas ${ }^{2}$ generaron cambios en la morfología del lugar y recurrimos a la heterotopía para catalogar este fenómeno territorial (Figura 1) como un lugar que se ha insertado en otro, produciendo una nueva concepción de la realidad. Dicho concepto es trabajado por autores como Foucault (2008), Hetherington (1997), Sánchez (2008), Saldanha (2008), West-Pavlov (2009), Topinka (2010) y García (2014). En el caso que nos ocupa, las heterotopías que predominan en este territorio son el monocultivo de la caña de azúcar y las relaciones fácticas de su encadenamiento productivo como despensa hortofrutícola que otorga nuevos órdenes a las dinámicas socio-políticas y las prácticas económicas en el Norte del Valle del Cauca.

Introducimos otros elementos para comprender el modelo territorial que muestra la estrecha relación sistémica entre procesos económicos, históricos y socio-culturales según (Raffestin, 1980, p. 52) tales como “[...] El territorio es concebido como espacio político por excelencia y esto determina en gran medida -la morfología [...] y la lógica de la estructura-", además la tesis que concebimos fue que toda territorialización es política y que en tanto política es un acto de poder y da cuenta de cómo se ha configurado el lugar; con Giménez (2000, p. 21) conceptuamos que todo territorio $^{3}$ tiene su morfología y que los actores empresariales durante el

\footnotetext{
${ }^{1}$ El lugar corresponde al municipio de Zarzal, unidad espacial en su naturaleza de carácter urbano que hace parte del corredor vial de la Doble calzada Buga-Tuluá-La Victoria y la Concesión Vial del Café, ruta nacional 29. Su fundación data de principios del siglo XIX, durante la época de la colonia fue un cruce de caminos entre las diversas campañas militares e independentistas, pero antes de ello fue el asentamiento de comunidades indígenas gorrona, daimará y pijaos en las riberas del río La Paila; en 1972, figura en el ámbito nacional por la huelga de los trabajadores del Ingenio Riopaila y las implicaciones laborales, económicas y culturales, Sánchez (2008, pp. 34-57).

${ }^{2}$ Tales como: transformación del jugo de la caña en azúcar, alcohol carburante, mieles, fabricación de dulces, la comercialización de frutas y otros productos en la industria de alimentos, ganadería, entre otros.

${ }^{3}$ Giménez (2000, p. 21) "territorios interiores considerados en diferentes escalas (v. gr. Lo local, lo regional, lo nacional, etcétera) siguen en plena vigencia, con sus lógicas diferenciadas y específicas, bajo el manto de la globalización, aunque debe reconocerse que se encuentran sobredeterminados por ésta $\mathrm{y}$, consecuentemente, han sido profundamente transformados en la modernidad. Hay [...] lecciones que, pese a todo, debemos aprender de los teóricos neoliberales de la globalización. Los territorios se transforman y evolucionan incesantemente en razón [a la] mundialización geopolítica y geoeconómica.
} 
siglo XX han realizado cambios que responden a intereses de expansión de las lógicas comerciales. Aunque Halbwachs (2004, p. 5) considera que "el pensamiento social es básicamente una memoria y que todo su contenido está hecho de recuerdos colectivos, pero sólo permanecen presentes en la sociedad esos recuerdos que la sociedad trabajando sobre sus marcos actuales puede reconstruir", ello nos sirvió para evidenciar los cambios a partir de las implicaciones de los discursos que dan materialidad a las representaciones de las formas nombradas del territorio.

En el trabajo de campo objetivamos recursos que mutan de ser naturales a lo producido (Raffestin, 1975, p. 195), además de la influencia de las relaciones establecidas con el espacio social que genera flujos, mercantilización y asimetrías, porque en la caracterización de los 20 actores empresariales, evidenciamos que territorializan y a través de su legado industrial transforman el territorio en una heterotopía. En la etnografía realizada, presentamos los vínculos de una sociedad, (Bourdieu, 1995) las relaciones y las conexiones entre identidad con los procesos socioeconómicos; según Elias (1987), para entender los efectos económicos y sus propósitos es indispensable analizar las conexiones que se establecen en un contexto global y no un sólo factor. Sin embargo, tal como lo plantea Sassen (2007), es necesario dar nombre a ciertas condiciones nuevas que se hacen visibles gracias a las alteraciones en las configuraciones del territorio.

Recurrimos en primera instancia al análisis de los discursos para comprender las representaciones territoriales de nueve actores sociales, entendiendo de paso, sus esquemas de acción en los procesos de transformación, los nexos, la integración y la articulación que ha generado emplazamientos de distinto orden socio-económico, en un segundo momento a las evidencias empíricas encontradas en la etnografía (Urdinola, 2011 y 2014) y las implicaciones de las prácticas que influyen en las representaciones de la identidad. Nos apoyamos en (Bourdieu, 2002, p. 22) para definir "las representaciones [...que] significan elaborar [...] clases teóricas lo más homogéneas posibles desde las perspectiva de [...] las prácticas y de todas las propiedades que resultan de ellos", además conceptualizamos el lugar partiendo del sentido territorial de los practicantes en un orden individual y colectivo, centramos el análisis diacrónico en la relación espacio-temporal y en las disposiciones de cambio de los actores producto de su rol como sujetos políticos actuantes y en la dimensión económica para la caracterización de un territorio (Di Méo, 1998, p. 27), considerando que las prácticas de los actores empresariales y sus

[Los] territorios siguen siendo actores económicos y políticos [...] y siguen funcionando como espacios estratégicos, como soportes privilegiados de la actividad simbólica y como lugares de inscripción de las 'excepciones culturales', pese a la presión homologante de la globalización". 
relaciones están dadas por los recursos en función de la optimización de los beneficios esperados, los intercambios y la necesidad de expansión, influenciando las representaciones.

El período de estudio fue de 1990 a 2010, allí se contrastaron datos de la realidad empírica con el corpus teórico de Raffestin (1980), Elias (1987), Bourdieu y Wacquant (1995), Hetherington (1997), Foucault (2008), Giménez (2000), Bourdieu (2001 y 2003), Di Méo y Buléon (2005) y Sassen (2007). Evidenciamos en el trabajo de campo las representaciones territoriales de los actores sociales sobre las tensiones de cómo un espacio social es practicadovivido y cómo las prácticas económicas durante el siglo XX superponen otros lugares en la morfología territorial del municipio de Zarzal; objetivamos dimensiones socio-políticas y los efectos de las heterotopías en un modelo territorial complejo y las manifestaciones territoriales de los actores empresariales que son reconocidos por su liderazgo económico en la construcción de equipamientos, redes y alianzas.

\section{Diagnóstico}

Para caracterizar el municipio de Zarzal, recurrimos a fuentes documentales en las décadas 1990 a 2010, al trabajo etnográfico en los años 2011 a 2014. Una vez sistematizadas, determinamos las representaciones territoriales centrándonos en la disposición y las prácticas económicas, la influenciaenlatransformación delamorfologíalugareñaenlareconfiguración y los emplazamientos de toda índole: movimientos económicos, sociales y culturales, como la agricolización ${ }^{4}$, la cañarización y la pecuarización que con el transcurrir del tiempo contribuyó de manera particular al paisaje productivo. Sin embargo, después de realizar el recorrido presencial por Zarzal, se halló que las representaciones territoriales de los actores sociales indican que empresas como el ingenio azucarero, haciendas frutícolas, hatos lecheros, trapiches paneleros y fábricas de dulces han influido con su acciones en cambios de la morfología, insertando otras dinámicas; observamos grandes extensiones de tierras cultivadas con caña de azúcar y frutales así como diversos acondicionamientos en las redes de transporte y según las fuentes primarias consultadas hubo intermediación en la construcción de barrios en el área urbana y rural, edificando equipamientos dentro de sus complejos industriales, constituyendo heterotopías en este lugar.

\footnotetext{
${ }^{4}$ Es decir, la diversidad de cultivos establecidos y prácticas productivas, que fueron inventariadas en el contexto local, producto del análisis de fuentes primarias y secundarias.
} 
En el análisis de la territorialización ${ }^{5}$, evidenciamos la relación directa de los actores empresariales con el lugar, un ejemplo son los ejes viales y los paisajes productivos. Lo anterior se obtuvo desde la concepción de la visión de lo regional y los vínculos comerciales para comprender las representaciones territoriales; a partir de la clasificación de información recolectada encontramos cómo un escenario es reconocido y delimitado a través del establecimiento de relaciones que centralizan lugares claves en la localidad y asumimos una postura teórica en los estudios territoriales y la escuela neoinstitucionalista de la economía en torno a la relación del objeto de estudio y el fenómeno abordado que permitió, en esta perspectiva disciplinar, develar el modelo funcional regional que es establecido en los vínculos del lugar con las prácticas transgeneracionales de los actores empresariales que influenciaron la estructura del territorio.

En las entrevistas aplicadas a los actores sociales evidenciamos cómo éstos representan los procesos socioeconómicos, los vínculos adquiridos en el lugar, los cambios poblacionales, las prácticas de los actores empresariales en el municipio y como ello contribuyó al giro económico, político y cultural durante el siglo $X X$, comprendiendo ello en los niveles multiescalares y la percepción de las relaciones políticas locales y nacionales al abarcar inteligibilidades que muestran la representación del lugar a partir de los procesos socioeconómicos. En la sistematización de los datos recopilados en campo identificamos las dinámicas funcionales-regionales, el liderazgo local, los vínculos y prácticas económicas de los actores empresariales, evidenciando su impacto en el lugar en donde "tienen la vocería en las relaciones que controlan un territorio". (A. H.: No. 3: 117, junio 2013).

\section{Metodología}

La construcción teórica del objeto de estudio permitió reconocer el espacio social, su sentido y apropiación (Halbwachs, 1938) develando las territorialidades, la representación de lo cotidiano y sus conexiones con el lugar (Lefebvre, 2006). Visibilizamos la experiencia colectiva en la estructura del poder dominante y su influencia en el acondicionamiento de los equipamientos.

\footnotetext{
${ }^{5}$ Fenómeno que refleja la influencia simbólica del poder de los actores sociales para fijar límites y generar actos de posesión y posición referente a las acciones en el lugar; estos hechos infunden jerarquía territorial en el comportamiento, delimitando y ejerciendo acciones visibles que marcan; en esta investigación se lee a partir de equipamientos realizados con participación influyente, reconocida por los pobladores como un símbolo de poder, instituyendo con sus nombres íconos emblemáticos o geosímbolos en el lugar. Algunos autores como Contreras (2002, p. 41) y Tapia (2009, p. 142) han realizado estudios que resaltan la importancia de estos geosímbolos para comprender las dinámicas rurales, los impactos y la simbología en función de la realidad empírica.
} 
Para comprender las lógicas de región contextualizamos a Tuluá y Cartago, municipios ubicados en el norte y el centro del Valle, los cuales presentan relaciones funcionales con Zarzal que centran los servicios financieros y los abastecimientos de insumos para los sectores de alimentos, textiles y construcción, recorriendo los lugares emblemáticos por medio de la objetivación no participante, allí sistematizamos las prácticas que influyeron en los cambios del lugar.

En Zarzal escogimos al grupo Caicedo González por ser referente de liderazgo e impacto económico, educativo y social de sus empresas el Ingenio Riopaila-Castilla, la Fábrica de Dulces Colombina, la institución educativa Hernando Caicedo y la Fundación Caicedo González. Según lo indagado en las entrevistas (Urdinola, 2013 y 2014) genera significación de identidad como "Zarzal, la tierra que endulza a Colombia", y en las últimas décadas dichas empresas accedieron a sistemas de equipamientos sofisticados (Figura 1).

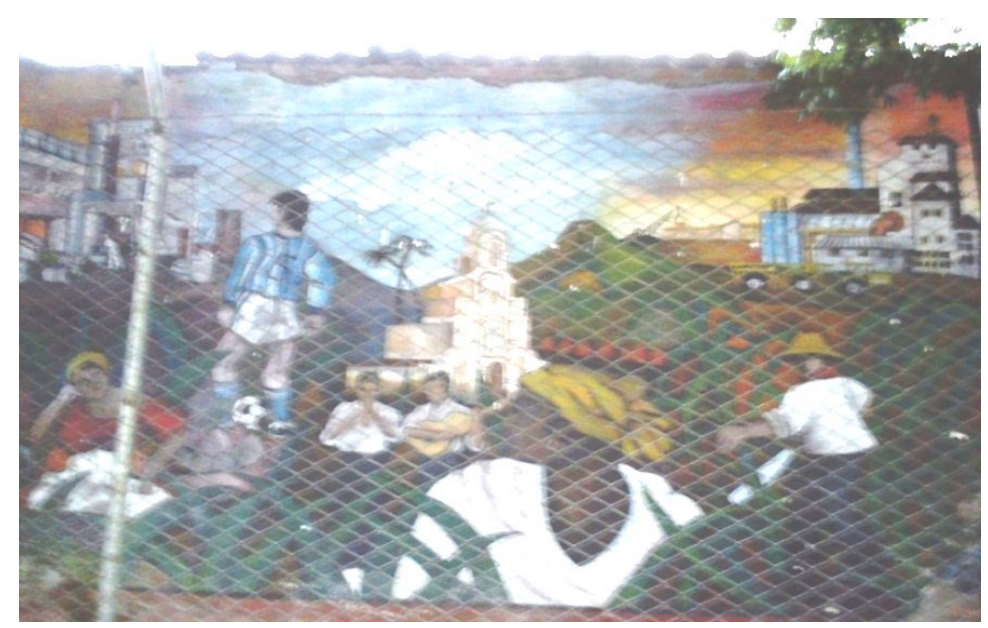

Figura 1. Mural La Paila, representación territorial. Registro fotográfico en exteriores, Institución Educativa Antonio Nariño.

Fuente: Urdinola (2014).

El análisis de las entrevistas y la etnografía determinó el valor de uso, las lógicas de la construcción de los equipamientos con las consecuencias en la apropiación identitaria y cultural de otros grupos migrantes, debido a la cantidad de mano de obra atraída por las oportunidades laborales para desempeñar los oficios de campo y de fábrica y por la herencia de un 
modelo de hacienda feudal predominante en el siglo XIX en las actividades productivas de la caña que hoy conocemos como agroindustria, que aún atrae a pobladores oriundos de departamentos como Nariño, Chocó, Cauca y otros lugares del país.

En las fuentes secundarias encontramos la funcionalidad de los nexos local-regional de las dinámicas comerciales del centro-norte del Valle, la lógica de intercambio entre productores y consumidores que dinamizaron los nexos de la economía local con la agricultura intensiva, los sectores comercial y de alimentos procesados que en los últimos veinte años ha sufrido cambios vía globalización económica, reduciendo el área cultivada en lo que respecta a la diversidad de oferta alimentaria (hortalizas, verduras y algunos frutales) que antes de los años 90 fuera potencia agrícola en área cultivable de productos como cebolla junca, algodón, girasol y trigo, los cuales desaparecieron en el período de estudio (Figuras 2 y 3 ).

Lo anterior, sumado a la disminución de producción de frijol-plana, sorgo y soya, pasaron de manera notable la sumatoria de los tres ciudades más importantes del centro y norte del Valle del Cauca, en su orden: Tuluá, Zarzal y Cartago de 172.171 toneladas en 1990 a 12.612 en 2009 y para los trece municipios que constituyen el centro y norte del Valle del Cauca ${ }^{6}$ se observaron cifras en descenso que al compararlas departamentalmente en el mismo período de análisis se encontró que de 305.046 pasa a 20.352 toneladas de acuerdo con anuarios estadísticos de los años 1990 y 2009, es decir, que el porcentaje de representatividad cambia de $56,44 \%$ a $61,96 \%$ para dichos municipios (Figura 3).

Con el cultivo permanente del café ocurre un hecho particular, al ser uno de los de mayor extensión mantiene su área en casi todos los municipios, desapareciendo en sólo uno de ellos; así mismo se nota que ocurre un descenso en el departamento de 110.347 en $1990^{7}$ a 63.816 toneladas en 2009 permaneciendo el 57,83\% de la producción en este último año. Paralelo a la situación evidenciada otros cultivos emergentes como la pitahaya, tanto a nivel departamental y municipal, presenta producciones bajas, observándose en los años 1990 y 2009 por debajo de mil toneladas/ año (Figuras 2 y 4 ).

\footnotetext{
${ }^{6}$ Alcalá, Andalucía, Ansermanuevo, Argelia, Bolívar, Bugalagrande, Caicedonia, Cartago, El Águila, El Cairo, El Dovio, La Unión, La Victoria, Obando, Roldanillo, Sevilla, Toro, Tuluá y Zarzal.

${ }^{7}$ La cantidad aportada en el 1990 de los trece municipios a dicha producción es de 77.266 toneladas, lo que corresponde al 70\% departamental, el cual en 2009 sigue en aumento dicho porcentaje con un $73,2 \%$, aunque el nivel de producción presenta una baja de 46.732 toneladas.
} 
Representaciones territoriales y heterotopías en Zarzal (Valle del Cauca - Colombia) |

\begin{tabular}{|c|c|c|c|c|c|c|c|c|}
\hline \multirow{3}{*}{ MUNICIPIOS } & \multicolumn{8}{|c|}{ PRODUCCIÓN CULTIVOS PERMANENTES } \\
\hline & \multicolumn{2}{|c|}{ Caña de azúcar (1) } & \multicolumn{2}{|c|}{ Caña panelera (1) } & \multicolumn{2}{|c|}{ Plátano } & \multicolumn{2}{|c|}{ Cebolla junca } \\
\hline & 1990 & 2009 & 1990 & 2009 & 1990 & 2009 & 1990 & 2009 \\
\hline VALLE & 10239864 & 23141404 & 454028 & 369568 & 301582 & 162328 & 7969 & \\
\hline TOTAL MUNICIPIOS & 2064738 & 6004074 & 229803 & 169440 & 209844 & 116218 & 329 & \\
\hline Alcalá & & & & 6344 & 14268 & 1071 & & \\
\hline Andalucía & 145854 & 306499 & 934 & 33579 & 2158 & 560 & & \\
\hline Ansermanuevo & 93082 & 286264 & 21814 & 5031 & 12879 & 4966 & & \\
\hline Argelia & & & 1610 & 3366 & 6647 & 16080 & & \\
\hline Bolívar & 9938 & 57395 & 92372 & 22176 & 1239 & 4238 & 30 & \\
\hline Bugalagrande & 518009 & 1023650 & 3695 & 22248 & 7300 & 2320 & & \\
\hline Caicedonia & & 11795 & 5428 & 1350 & 45641 & 16359 & & \\
\hline CARTAGO & & 308771 & 875 & 4032 & 9221 & 1206 & & \\
\hline El Águila & & & 1015 & 5400 & 17964 & 6333 & 12 & \\
\hline El Cairo & & & 19025 & 1771 & 2689 & 31366 & & \\
\hline El Dovio & & & 2192 & 14783 & 3274 & 400 & 12 & \\
\hline La Unión & & 121040 & 2380 & 760 & 1769 & 173 & & \\
\hline La Victoria & 43268 & 128155 & 8418 & 8282 & 3825 & 1680 & & \\
\hline Obando & 5212 & 357605 & 2442 & 1940 & 6344 & 2700 & & \\
\hline Roldanillo & & 192784 & 646 & 2232 & 797 & 456 & 23 & \\
\hline Sevilla & & & 7040 & 11520 & 45680 & 12249 & & \\
\hline Toro & & 125583 & 2004 & & 3120 & 2830 & 12 & \\
\hline TULUÁ & 597319 & 959829 & 2740 & 4032 & 16622 & 3728 & 240 & \\
\hline Ulloa & & & & & 6209 & 4185 & & \\
\hline Versalles & & & 38828 & 19044 & 2160 & 3220 & & \\
\hline ZARZAL & 652056 & 2124704 & 16345 & 1550 & 38 & 98 & & \\
\hline no se observa & & & & & & & & \\
\hline
\end{tabular}

Figura 2. Cultivos permanentes en toneladas, centro y norte - Valle del Cauca. Fuente: Anuarios estadísticos Valle del Cauca, años 1990 y 2009.

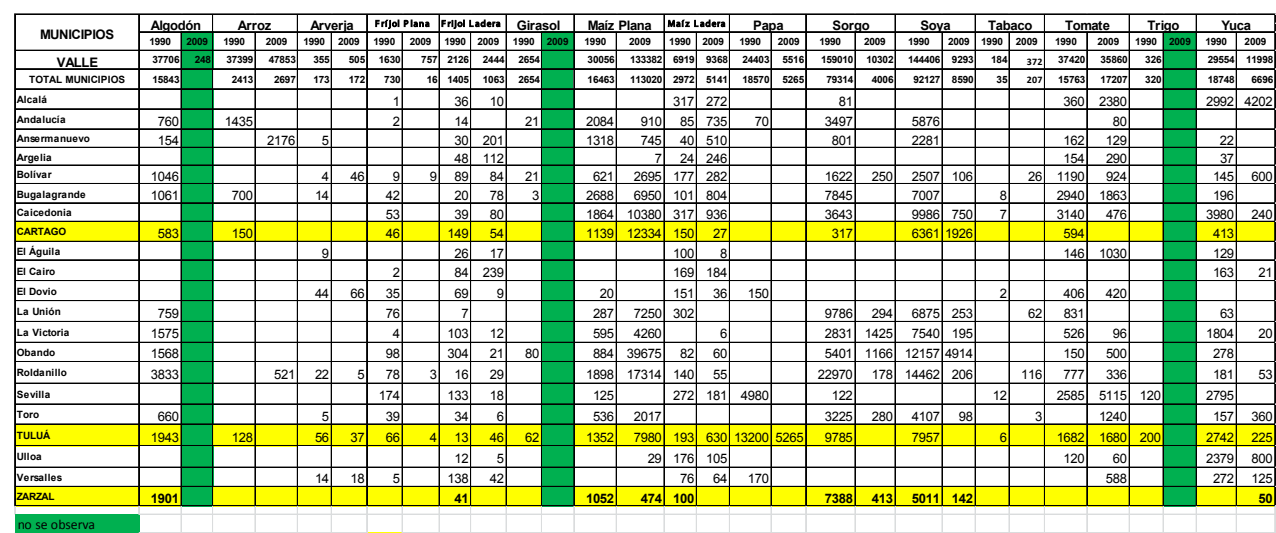

Figura 3. Comportamiento producción cultivos, norte y centro- Valle del Cauca. Fuente: Anuarios estadísticos Valle del Cauca, años 1990 y 2009. 


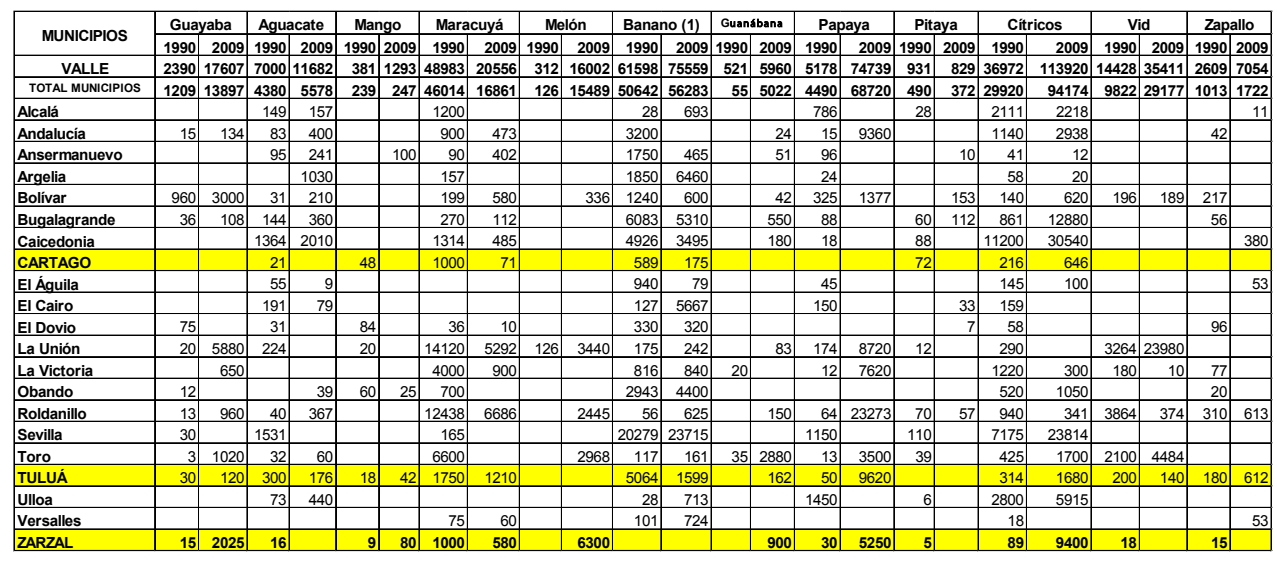

Figura 4. Producción cultivos frutales, norte y centro- Valle del Cauca. Fuente: Anuarios estadísticos Valle del Cauca, años 1990 y 2009.

En el caso de los cultivos permanentes de las cañas de azúcar y de panela ${ }^{8}$, observamos en las décadas de 1990 a 2009 un hecho particular en la producción vs. área cosechada, en el departamento en la primera se presenta un aumento al pasar en el 90 de 10.239 .864 a 23.141.404 toneladas en 2009 y en la segunda un descenso en 1990 de 454.028 a 369.568 toneladas en el 2009. Sin embargo, en el caso de la caña de azúcar se fortaleció en cuatro municipios que para la década de 1990 no se evidenciaba (Figura 2). Una de las causas que influenció las dinámicas de rendimiento entre la producción y la comercialización obedece a la implementación de tecnología que posibilitó mayores transacciones comerciales y especialización del terreno (Gobernación Valle del Cauca, 2006, p. 40).

En la caracterización territorial empleamos un análisis diacrónico que destaca 3 hitos representativos de Zarzal a manera de atestiguar y contrastar los cambios evidenciados en dicho lugar:

1. En la infraestructura vial se evaluó la cronología de la Construcción de la Concesión Buga-Tuluá-La Paila, inaugurada los primeros tramos de la doble calzada en 1996 y que incrementó los flujos del transporte en la zona; en 2008 se firmó continuidad hasta La Victoria para adherirse con la Autopista del Café -Manizales-Armenia- o Ruta Nacional 29 (Figura 5).

\footnotetext{
${ }^{8}$ La cantidad aportada en el caso de la caña de azúcar en 1990 de los trece municipios a dicha producción es de 2.064.738 toneladas, para 2009 aumenta esta cifra a 6.004.074 toneladas y en la caña panelera el comportamiento es distinto; disminuye de 229.803 toneladas en 1990, a 169.440 en 2009, respectivamente.
} 
Representaciones territoriales y heterotopías en Zarzal (Valle del Cauca - Colombia) |

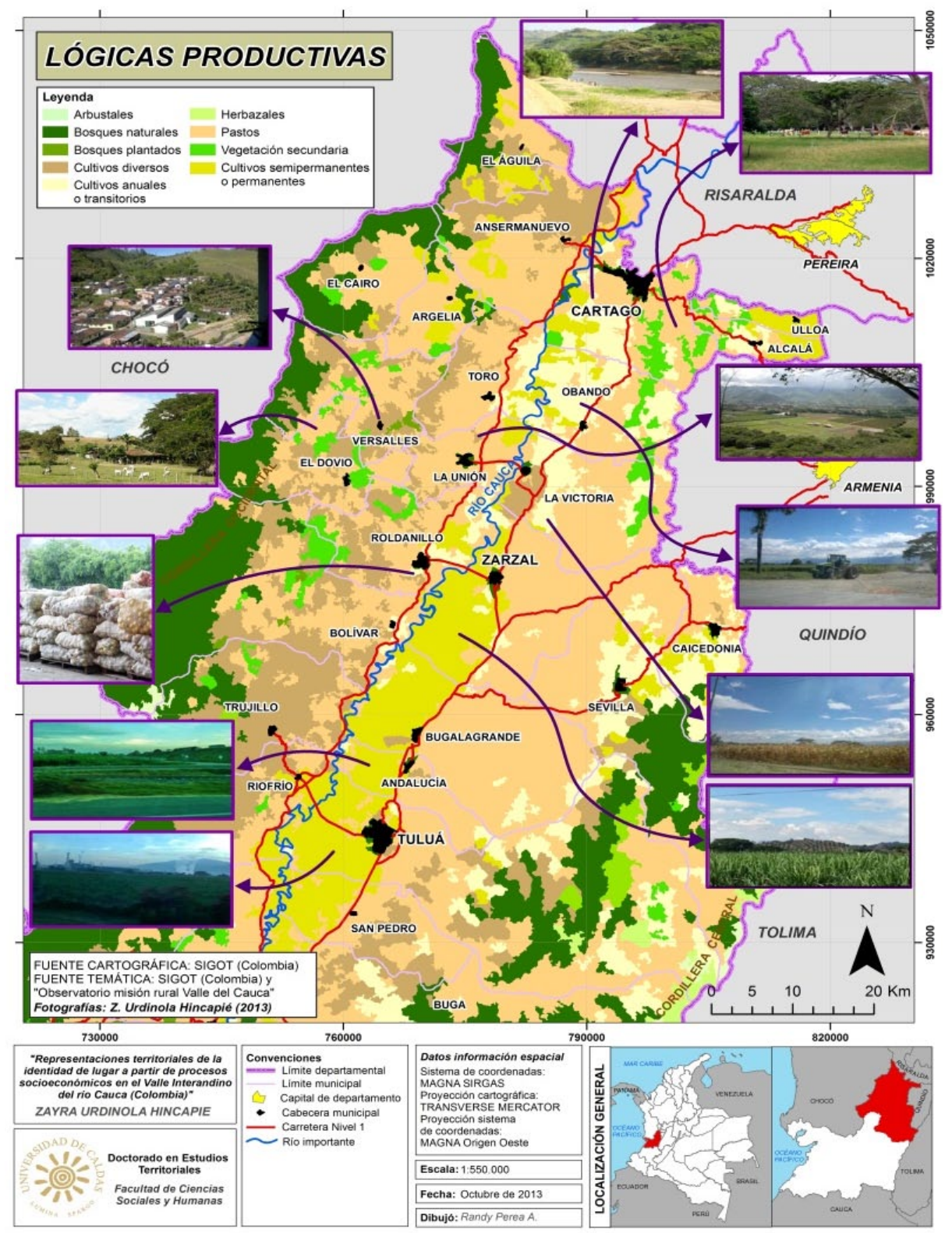

Figura 5. Mapa centro-norte, Valle del Cauca. Fuente: Urdinola (2014). 
2. En las actividades productivas se construye un mapa de la región centro-norte (Figura 5), que evidencia nexos entre las prácticas económicas y las relaciones comerciales (Urdinola, 2011-2014), las lógicas productivas y cómo los geosímbolos ${ }^{9}$ ubicados estratégicamente en el territorio constituyen prácticas de poder de los actores empresariales; al igual que sucede con los nombres otorgados a éstos, se esclareció la funcionalidad de los sistemas de infraestructura vial, los cuales fueron dispuestos de acuerdo con las necesidades comerciales.

Tabla 1. Equipamientos municipio Zarzal.

\begin{tabular}{|c|c|}
\hline CENTRALES & DESAPARECIDOS \\
\hline $\begin{array}{l}\text { Empresas 1. Ingenio Riopaila (caña) } \\
\text { 2. Colombina S.A. (dulces) } \\
\quad \text { 3. Hacienda el Medio, El Garcero } \\
\text { (ganadería) } \\
\text { Escuela Colombia, Colegio Antonio Nariño } \\
\text { Hacienda el Guavito } \\
\text { La iglesia } \\
\text { Estación gasolina, dispensario, bomberos, } \\
\text { escuela, gimnasio, hotel, teatro y banco en } \\
\text { Riopaila }\end{array}$ & $\begin{array}{l}\text { Cultivo y centros de acopio: } \\
\text { Café } \\
\quad \text { Maíz } \\
\text { Algodón } \\
\text { Estación del ferrocarril } \\
\text { Costalería } \\
\text { Matadero }\end{array}$ \\
\hline
\end{tabular}

Fuente: Urdinola (2014), a partir de instrumentos entrevista de trabajo de campo No. 1: 27, 28, 33, 68, 69, $103,140,142,148,223,275,276,216,229,253$ y 301 junio 2013 y etnografía territorial años 2011-2014.

3. Uno de los efectos espaciales caracterizados en la tabla 1 es la transformación de las actividades productivas que han impactado los lugares en el norte del departamento, tal es el caso de la expansión de la frontera productiva de los cultivos permanentes, que cada vez requiere mayor concentración de tierras para la caña de azúcar y frutales, las actividades ganaderas, los centros de procesamiento y las fábricas, constituyéndose éstos últimos en epicentros de transformación de materias primas e insumos; al igual los hatos lecheros y avícolas que han desplazado otros productos alimenticios y de paso la fauna y flora de dichas áreas. Además, clasificamos las alianzas y prácticas productivas dominantes, la territorialización del poder y las representaciones sobre diversos órdenes que influencian el lugar.

\footnotetext{
${ }_{9}^{9}$ Son imagos, "simbología del poder" que imponen la circularidad del reconocimiento de los intercambios económicos y sociales que instituyen los actores, sujetos que ocupan una posición en el territorio, en este caso hay apropiación de estos por el lugar desde las prácticas empresariales.
} 
Otros aspectos del análisis espacial que aportan elementos complejos a considerar en el control territorial fue el fenómeno del narcotráfico, las identidades transitivas y su influencia en las relaciones económicas de facto (Sánchez, 2008), los cuales asociamos con el concepto de heterotopías porque en el contexto rural, en especial en el norte del Valle y según lo aportado por los actores y fuentes secundarias, esto propició condiciones favorables a estas otras prácticas productivas ilícitas, lo que ocasionó cambios drásticos en las morfologías territoriales tales como deforestación de grandes extensiones de bosque nativos para cultivar coca o instaurar laboratorios para su procesamiento, entre otros.

El cuestionario semiestructurado se utilizó para reunir la información primaria con variables definidas en el análisis de los discursos y las representaciones, nos centramos en los cambios físicos que para este caso permitió evidenciar las morfologías en el lugar, el papel de las heterotopías y los efectos generados en el territorio. Las actividades realizadas para visibilizar los fenómenos insertos en la zona de estudio, fueron:

- Clasificación de los discursos de líderes cívicos, comunitarios y políticos cesantes, entendidos para el estudio como los actores sociales, para comprender las prácticas de reconfiguración territorial de los actores empresariales.

- Análisis de los discursos en el reconocimiento de los procesos de territorialización y sus evidencias socio-espaciales de los cambios del territorio, por medio de elaboración de cartografía, ubicando geosímbolos locales, y se caracterizó el modelo territorial en la región centro y norte del Valle del Cauca.

\section{Resultados}

Las relaciones políticas están mediadas por la territorialidad e influencian la identidad y los procesos socioeconómicos dados en el espacio social, lo cual interviene en la morfología del territorio. En este sentido, Di Meo (1998, pp. 6 y 38) dice que la territorialidad simboliza una importante socialización y visibiliza un espacio determinado por las prácticas cotidianas, las bases simbólicas de lugares que forman territorio identitario, manifestándose en la movilización social por medio de la reconstrucción del recorrido simbólico de los actores que dejan sus marcas en el lugar. Como habíamos señalado, las heterotopías evidencian relaciones practicadas de poder, por tanto, las territorializaciones reflejadas en los equipamientos con sus geosímbolos construyen otros territorios insertos en ese mismo territorio. 
A partir de la representación territorial realizada, proporcionamos información de los actores empresariales sobre los efectos configuracionales en la estructura del lugar. Para Hetherington (1997, p. 22): “las prácticas espaciales se asocian con la producción de distintos espacios y relaciones sociales con la producción capitalista y la reproducción. La representación del espacio cómo producto de las ideologías hegemónicas asociadas con el espacio producido"; y evidenciamos las heterotopías (Foucault, 2008, pp. 39-62) y ratificado por West-Pavlov (2009) como un efecto de las organizaciones que han condicionado y generado otros órdenes en la vida cotidiana. En Zarzal, según datos de la Cámara de Comercio de Tuluá (2010, pp. 9 y 14), están asentadas 1.483 empresas y las que más aportan empleo son del sector de la industria manufacturera con el 52,4\% y 3.836 empleos en 2009, destacándose el Ingenio Riopaila-Castilla y la fábrica de dulces Colombina, entre otras.

Utilizamos la sintaxis espacial ${ }^{10}$ (Reynoso, 2010) para develar el "sentido práctico" (Bourdieu, 2001), al sintetizar los patrones de los arraigos territoriales, a partir de un modelado territorial que representa, a través de un mapa axial ${ }^{11}$ los imagos, las heterotopías y los lugares practicados que marcan las relaciones, los usos y las prácticas (Figura 6).

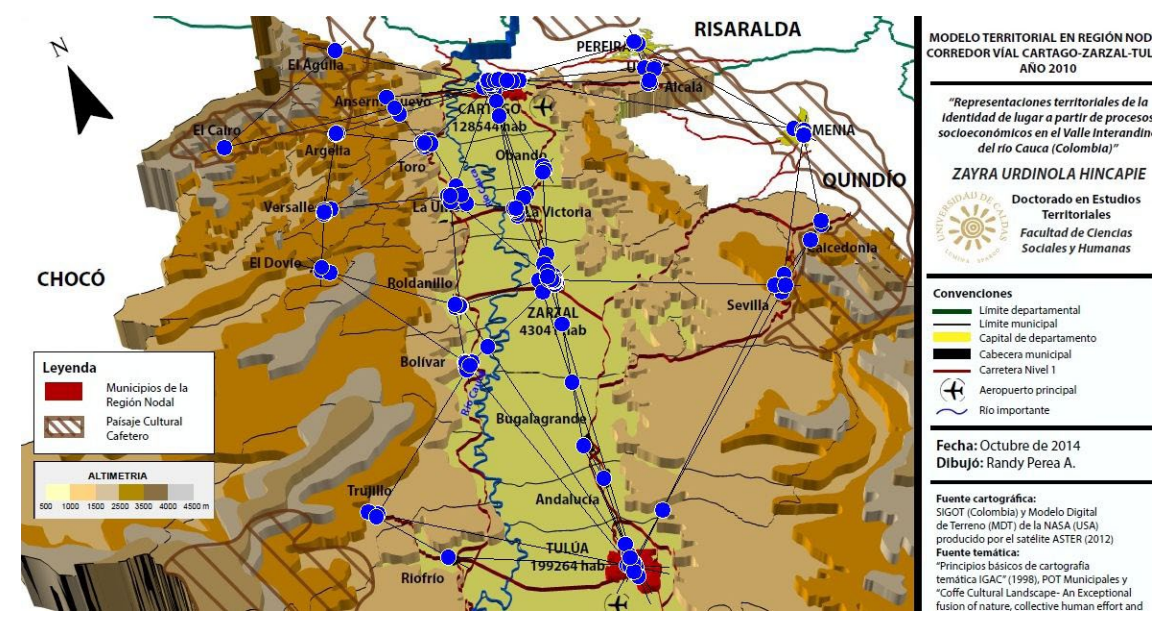

Figura 6. Modelo relacional, mapa axial- centro norte, Valle del Cauca.

Fuente: Urdinola (2015), elaborado a partir de software AJAX LIGHT versión 1.02 july 2005, disponible: http://www.casa.ucl.ac.uk/software/ajax.asp

\footnotetext{
${ }^{10}$ Para diseñar el modelo comprensivo tipo explicativo con énfasis en las relaciones territoriales y las morfologías, localizando en el territorio los nexos funcionales entre nodos en los municipios del Centro y Norte del Valle del Cauca.

${ }^{11}$ El gráfico axial tiene 248 arcos y 59 nodos. Esto da una proporción de 4,2 arcos a los nodos, muestra los vínculos entre los puntos medios de cada línea axial, ilustra un indicador de la densidad de líneas en la medida en que tiene sentido espacial.
} 
Aportamos otros determinantes ubicados desde el análisis del discurso para la interpretación de los imagos e infografías ${ }^{12}$ en el territorio y con ello resignificar el lugar. En este apartado los actores reconocieron en la entrevista su valor de uso y los cambios sufridos en el municipio que insertan marcas presentando un fenómeno espacial; a continuación, relacionamos los hallazgos clasificados en tres categorías: las heterotopías, los desequilibrios económicos derivados y las representaciones territoriales frente a la dinámica migratoria producto de la oferta de empleo:

- Heterotopías:

- “Contribución del Ingenio Riopaila a la consolidación del corregimiento: campamentos para los corteros de caña (La Luisa, Alizal)... que hoy son campo de caña". "Construyó tres barrios [...], luego en [...] Zarzal, Roldanillo, Tuluá y un modelo educativo para mejorar calidad de vida de trabajador y familia: -primaria, bachillerato y -alianza con SENAUniversidad: [...]". (Z. H.: No. 2: 9, 21, 22, 26 y 31, junio 2013)

-[...] venta de productos [...] para los hijos de los trabajadores del Ingenio Riopaila en la Cooperativa, “...tengo vestido, tengo techo" “[...] ya después la estructura urbana del corregimiento se complementó con la ayuda a las familias de los trabajadores para que se asentaran [...], luego se crearon los barrios Santa Cecilia, Hernando y Belisario Caicedo, "los últimos por intervención de Riopaila". (A. H.: No. 3: 178 y 180, junio 2013 y Z. H.: No. 2: 22, junio 2013).

-Rol protagónico: “...La Paila [corregimiento de Zarzal] sin la familia Caicedo: sería casas de palmito..., dos o tres matas de plátano..., El vinito..., Las verduritas"... "el líder no es sólo para conseguir obras, sino cambios culturales". (A. H.: No. 3: 72, 104 y 109, junio 2013)

- Representaciones territoriales de nativos residentes frente a la migración de grupos poblacionales atraídos por la oferta de empleo:

-Sobre la etnia negra que migró a La Paila: “[...] llegó atraída por ensanche azucarero en la década de los 60". "[...] ha evolucionado en otra generación y hoy hay un nuevo tipo [...]: un híbrido: el negro de aquí es distinto: es fiestero, su color de piel, en la forma de hablar al costeño, gozón [...]". (A. H.: No. 3: 43, 48, 49, 52, 54, 61, 62 y 65, junio 2013)

-Contratación de mano de obra: “desde los 50, se atrae nuevas culturas para mano de obra en las empresas de Ingenio Riopaila y Colombina

\footnotetext{
${ }^{12}$ Entendida como la representación del territorio y la imagen que evoca su espacialización, es la forma de etnografiar la realidad, el reordenamiento, las instituciones que condicionan la morfología de los espacios desde las dimensiones (Raffestin, 1980, p. 129).
} 
S.A.: Nariño, Cauca, Chocó, Tolima, otros municipios (Buenaventura, Tuluá, Bugalagrande, Roldanillo, Zarzal, Sevilla y La Marina)”, “...los que han venido, ya todo está hecho" (D. L.: No. 1: 84, 85, 86 y 245, junio 2013). "Riopaila contrató aviones de Tumaco, motonaves de las costas de: Nariño, Cauca, Valle, Chocó, para la huerta de trabajo: en la caña". (A. H.: No. 3: 43 y 44, junio 2013).

- Desequilibrios socioeconómicos, como efecto de las heterotopías:

-Modificación en la conectividad vial: "Cambios en el trazado de la carretera Panamericana y aquí cambió... era doble calzada y se construyó par vial". (A. H.: No. 3: 21, junio 2013)

-Prácticas de control de la funcionalidad del modelo urbano-rural: "A las empresas no les conviene que el corregimiento progrese y [se] convierta en municipio: porque los impuestos se le incrementan". (D. L.: No. 1: 325 y 326, junio 2013)

-Pérdida de identidad: "La Paila se conocía como punto de encuentro (puerto terrestre), era el Ombligo de Colombia, hoy día, ya no" (...) “La Paila, [no es el] Juanchito del Norte del Valle". (A. H.: 3: 22, 27, 29, 30 y 68, junio 2013)

Sin embargo, pese a las heterotopías reconocidas y los procesos socioeconómicos develados en el análisis del discurso (Bourdieu, 2001), a partir de la ejecución de las ocho entrevistas a profundidad y una biográfica no estructurada (Urdinola, 2013), recopilamos los testimonios agrupados en la figura 6, que dieron cuenta de la percepción, acción, apropiación y reconocimiento de la realidad y el sentido territorial de la identidad, precisamos las propiedades objetivadas en el territorio y las actividades económicas y subjetivas como el sentimiento de pertenencia (Bourdieu, 2001).

Contrastamos los datos que integraron los procesos de investigación de acuerdo con Dietz (2014), Van Dijk (1996) y con la objetivación participante (Urdinola, etnografía territorial en trabajo de campo, 20112014), evidenciando la influencia de los intereses de actores empresariales dominantes en la estructura del lugar y analizando cómo es el proceso de construcción de la representación simbólica de la lógica empresarial en la estructura del modelo ocupacional.

En el análisis del discurso y la interpretación de la historia de La Paila, principal corregimiento de Zarzal, constatamos la heterotopía en dicho escenario y evidenciamos cambios en la estructura urbana que han sido producto de la intervención de los actores empresariales, generandoespacios específicos acondicionados a las necesidades de expansión; aunque hay 
excepciones de acuerdo con la etnografía realizada, que evidencia lugares que se salen del orden impuesto, en este caso, el parque principal de la localidad, aunque cuenta con el busto de Hernando Caicedo, es concebido desde el sentir de los pobladores como un espacio social dedicado los fines de semana para el goce, "la rumba" y el disfrute, para tomar "los tragos de aguardiente" y escaparse de lo laboral.

Las heterotopías encontradas prueban la existencia de escenarios y equipamientos que territorializan el poder de los actores empresariales ejemplo de ello son: la unidad deportiva; los barrios Santa Cecilia, Belisario y Hernando Caicedo; adecuaciones al parque principal y a la iglesia; construcción de acequia del río La Paila y bocatoma para el tratamiento del agua que se utiliza en fábrica; edificación del centro comercial que alojó en sus instalaciones a la cooperativa de trabajadores; el colegio Hernando Caicedo; el Hogar infantil Santa Cecilia para niños menores de 5 años; el Instituto de Desarrollo para La Paila -IDLA- y la Escuela de Formación Obrera -EFO-. Dicho poblado a principios del siglo XIX fue reubicado hacia un sitio más cercano de las vías nacionales -carretera central- con la noción de sus líderes que este cambio les traería facilidades de toda índole.

Según Martínez (2010), los poderes de los actores generan diferentes manifestaciones que para el caso que nos ocupa son heterotópicas y a su vez penetran el sentido en las relaciones sociales establecidas; sólo traemos a colación algunos hechos que han contribuido significativamente a facilitar la construcción de una nueva concepción, elaborada a partir de las alianzas empresariales y aportes en el surgimiento de instituciones como el Hospital Municipal San Rafael, la Universidad del Valle sede Zarzal, la base militar Tesorito, entre otros. Además, cada año celebran festividades culturales que evocan y resignifican la evolución y la importancia empresarial en el corregimiento de La Paila.

Un efecto de las prácticas productivas es el monocultivo de la caña de azúcar que predomina en el área cultivable del centro hasta el norte del departamento, allí no hay diversidad de cultivos de pan coger como en otrora; autores como González (1959) y Ramos (1990) detallan la cronología de los hechos empresariales en el municipio. Relacionamos los datos anteriores con las entrevistas realizadas a profundidad (Urdinola, 2013), ubicamos la evolución económica de La Paila y el papel primordial de estos actores empresariales, lo contrastamos con los hallazgos del diario de campo y se ubicaron a los gestores de memoria (García, 2008 y 2014), para evidenciar las representaciones de nativos de La Paila, que en sus discursos evocan su historia de vida, precisando en el relato cómo el lugar se reconfiguró a lo que es hoy: "un sitio más urbanizado, lleno de gentes que no son de allí sino de [...] Nariño, Cauca y Chocó, esto como 
producto del empleo generado por las empresas, se apropiaron del lugar y se amañaron, hoy día hay muchos desconocidos, otros que se han ido y que nunca más volvieron"; evidenciamos algunos cambios en las disposiciones del territorio: "qué sería de Zarzal sin La Paila... sólo un cruce de caminos y que gracias a La Paila el municipio recibe los impuestos por rentas".

Presentamos aportes que condensan en la figura 8 el análisis relacional espacio-tiempo del norte del Valle en los años 1990 a 2010, a partir de varios esquemas de clasificación de la pertenencia sistémica de las relaciones teóricas de diversos elementos en el modelo territorial objetivado.

Tabla 2. Relación urbano-funcional, centro-norte Valle del Cauca.

\begin{tabular}{|c|c|c|c|c|}
\hline Municipios & $\begin{array}{l}\text { Lógicas de } \\
\text { producción }\end{array}$ & $\begin{array}{l}\text { Flujos } \\
\text { poblacionales }\end{array}$ & Flujos de capital & $\begin{array}{l}\text { Dinámicas de } \\
\text { organización } \\
\text { del trabajo }\end{array}$ \\
\hline TULUÁ & & & & Re 1 a c i ón \\
\hline ZARZAL & Comercialización & interr & Infraestructura & $\mathrm{un} \mathrm{id} \mathrm{a} \mathrm{des}$ \\
\hline CARTAGC & $\begin{array}{l}\text { Exportación } \\
\text { Importación }\end{array}$ & $\begin{array}{l}\text { Empleos } \\
\text { Informalidad }\end{array}$ & $\begin{array}{l}\text { vial } \\
\text { Equipamientos }\end{array}$ & $\begin{array}{l}\text { productivas, } \\
\text { poder político } \\
\text { y sindicatos }\end{array}$ \\
\hline
\end{tabular}

Fuente: Urdinola (2013). Adaptado según libro base Raffestin (1980).

- En cuanto al modelo territorial, fue confrontado con los presupuestos teóricos, presentamos algunos elementos caracterizados en las notas de campo y en la etnografía territorial (Urdinola, 2011-2014):Imagos destacados en el norte del Valle: "Roldanillo, tierra del alma", representación territorial predominante hacia la cultura, el principal atractivo cultural es el museo Rayo y el museo vial en la ruta ZarzalRoldanillo; encuentros internacionales de parapente y de mujeres poetas. En Zarzal, de acuerdo con la señalética turística ubicada en la entrada al municipio como "la tierra que endulza a Colombia" por sus empresas en este sector económico. En La Unión se encuentra todo lo relacionado con la vitivinicultura y otros frutales en el Parque Temático de La Uva. En Cartago, en la última década se ha posicionado este destino como la ruta comercial del bordado de corte artesanal y con capacidad de exportación que incluye a Ansermanuevo; otro aspecto destacado es la extracción manual y mecanizada de arena en el río La Vieja, una práctica económica que en los últimos tiempos ha mutado hacia otros campos para incentivar el fomento del turismo de aventura que incluye los paseos en canoa por dicho río. 
- Funcionalidades que demarcan la disposición económica, el dominio y el control territorial: la cañarización proporciona la mayor fuente de empleo de la zona centro-norte del Valle que incluye las unidades empresariales como: Ingenio Riopaila-Castilla (Zarzal), Ingenio San Carlos (Tuluá), Ingenio Risaralda (vía Balboa-La Virginia en Risaralda) con sembradío de caña en la zona rural de Cartago y la especialización de la Fábrica de Dulces Colombina S.A. (Zarzal).

- Presencia de heterotopías que muestran las lógicas de las prácticas económicas cuyas morfologías influencian los espacios sociales. Existen equipamientos céntricos que obedecen a la estructura del poder empresarial dominante: barrios, colegio, jardín infantil, entre otros.

- Actividades agropecuarias poco diversificadas y cultivos tecnificados de producción a escala que tienen mayor demanda por la compra de los productos e insumos nacionales e internacionales: caña de azúcar, café, plátano y frutales, al igual que la ganadería.

- Geosímbolos ubicados en zonas céntricas que reflejan la identidad y que marcan el territorio: escultura del ave Coclí, especie nativa endémica de Zarzal en vía de extinción. El Parque del Chontaduro en La Paila, busto de Hernando Caicedo en el parque principal de La Paila, mural ubicado en la institución educativa Antonio Nariño, que exhibe los principales referentes simbólicos en materia laboral: el ingenio, el obrero en este caso el cortero de caña, la pacora, los cañaduzales.

- Corredores viales y ferroviarios que obedecen a la lógica de conectividad global para la comercialización priorizado en necesidades de conexión, bajo las apuestas comerciales y productivas de las naciones.

\section{Conclusiones}

El territorio no es estático sino que es un espacio social complejo que refleja tensiones y conflictos presentamos las heterotopías y de paso materializamos la abstracción teórica del concepto en Zarzal, demostramos cómo éstas impactan los espacios sociales, producen y reproducen el sistema de lugares y reconfiguran identidades y de acuerdo con Bourdieu (2001), encontramos elementos para identificar el cómo se manifiesta un territorio y las jerarquías territorializadas que ejercen poder local al condicionar los ritmos cotidianos, visibilizando a dicho municipio en la concepción de que "las prácticas territoriales, marcan el lugar" por medio de la territorialización del poder (Urdinola, 2011-2014).

En la perspectiva relacional en la acción del sujeto, la afiliación, la identidad, las relaciones de fuerza, la inserción del discurso desempeña 
papeles estratégicos en la construcción y el cambio asumido por la sociedad ante los paradigmas derivados de los procesos culturales contemporáneos, la resignificación simbólica da lugar a nuevos reconocimientos pero también desde la corriente de los estudios territoriales podemos inscribir tendencias del análisis hacia la caracterización de categorías en la territorialidad y la territorialización, enriqueciendo el debate teórico-metodológico que implica desafíos conceptuales complejos que incluye otras miradas disciplinares y sobre todo la reflexividad, el análisis de la estructura y las relaciones de facto (Bourdieu, 2003).

Consideramos que las heterotopías constituyen un dispositivo de exclusión/fijación socio espacial, permitiendo la yuxtaposición de lugares en un espacio determinado para la interpretación de los diferentes órdenes espaciales: al lugar, el poder, el saber de los sujetos que emplazan con sus prácticas, ello remitió datos que se trabajaron en la construcción de las relaciones territorio/apropiación.

La concentración urbana del centro-norte del Valle del Cauca no depende de la densidad poblacional, aspectos como ser un corredor estratégico en lo económico, por la relación comercial jerarquizada en los Tuluá, Zarzal y Cartago, en comparación con los otros municipios de la altiplanicie se constituyen en ejes o epicentros que facilitan el intercambio y la conexión de redes funcionales urbano-rurales y morfologías representadas en imagos que como Lynch (1998), concebía incide en la configuración de focos de atracción y tensión; cambios físicos, tales como mayor extensión del área cultivable de la caña de azúcar e institucionalmente existen garantías y condiciones de mercado, con altos niveles de producción tecnificada que ha duplicado las transacciones comerciales y ocasionado un nuevo reordenamiento del territorio. Pero también desequilibrios territoriales que visibilizan las marcas de poder en el lugar de estudio, es decir que las actividades productivas de los actores empresariales influencian fácticamente el lugar y la globalización económica, posibilitando tendencias de libre comercio que constriñen el comportamiento de la economía local.

\section{Referencias bibliográficas}

Bourdieu, P. y Wacquant, L. (1995). Respuestas por una antropología reflexiva. México: Grijalbo. Bourdieu, P. (2001). Qué significa hablar. Madrid: Ediciones Akal S.A.

Bourdieu, P. (2001/2003). El oficio de científico. Ciencia de la ciencia y reflexividad. Curso del Collège de France 2000-2001, Trad. de Joaquin, J. 2001. Barcelona: Anagrama.

Cámara de Comercio de Tuluá. (2010). Comportamiento empresarial a diciembre de 2010, Observatorio socioeconómico. Recuperado de http://www.camaratulua.org/images/ comportamiento_empresarial/COMPORTAMIENTO_EMPRESARIAL_2010_12.pdf. [Marzo 13 de 2014]. 
Consejo Departamental de Estadística de la Gobernación del Valle-CODE-. (1990). Anuario estadístico del Valle del Cauca 1990-1991. Recuperado de ftp://ftp.colombiestad.gov.co/ books/LD_70129_1990_1991_EJ_2.PDF. [junio 21 de 2013].

Contreras, C. (2002). Espacio y sociedad. Estructuración espacial de un antiguo enclave minero. México: El Colegio de la Frontera Norte, Plaza y Valdés Editores

Dietz, G. (2014). La etnografía como artesanía. Desacatos, 46. Recuperado de http://www. scielo.org.mx/scielo.php?script=sci_arttext\&pid=S1405-92742014000300019\&lng=es\&tln $\mathrm{g}=\mathrm{es}$. [agosto30 de 2014].

Di Méo, G. (1998). Géographie sociale et territoires. Paris: Nathan.

Di Méo, G \& Buléon, P. (2005). L'espace social: lecture géographique des sociétés. Paris: Armand Colin.

Elias, N. (1987). La sociedad de los individuos, ensayos. Barcelona: Península.

Foucault, M. (2008). Topologías (dos conferencias radiofónicas). Revista Fractal, 13 (48). Recuperado de http://www.fractal.com.mx/RevistaFractal48MichelFoucault.html\#. [Abril 13 de 2013].

García, M. (2008). Memoria, territorio y otros simulacros. En: Nates-Cruz, B. (comp.) Conjuntos: miradas eurolatinoamericanas al estudio transversal del territorio. Lima: Instituto Francés de Estudios Andinos.

García, M. (2014). Los territorios de los otros: memoria y heterotopía. Cuicuilco, 21. Recuperado de http://www.redalyc.org/articulo.oa?id=35135452015> ISSN 1405-7778.

Giménez, G. (2000). Territorio, cultura e identidades. La región socio-cultural. En: Rosales, R. (coord.) Globalización y regiones en México. México: Editorial Miguel Ángel de PorrúaUniversidad Nacional Autónoma de México.

Gobernación del Valle del Cauca y Secretaría de Planeación. (2011). Anuario estadístico del Valle del Cauca 2008-2009. Subsecretaría de Asuntos económicos. Santiago de Cali, Recuperado de http://www.valledelcauca.gov.co/planeacion/publicaciones. php?id=19299. [enero 28 de 2013].

González, L. (1959). Zarzal, Charlas monográficas 1909-1959. Cali: Academia de Historia del Valle del Cauca.

Halbwachs, M. (1938). Individual Psychology and Collective Psychology. American Sociological Review, 5(3).

Halbwachs, M. (2004). Los marcos sociales de la memoria. España: Anthropos.

Hetherington, K. (1997). The Badlands of Modernity. Heterotopia and Social Ordering. New York: Routledge.

Lefebvre, H. (1986). La production de l'espace. Paris: Anthropos.

Lynch, K. (1998). La imagen de la ciudad. Barcelona: Gustavo Gili.

Martínez, J. (2010). Historia de los espacios, historia de los poderes: hacia una genealogía de la noción de espacio público. Tabula Rasa, 13. Recuperado de http://www.revistatabularasa. org/numero-13/indice.pdf. [enero 28 de 2012].

North, D. (1990). Instituciones, cambio institucional y desempeño económico. México: F.C.E.

Raffestin, C. (1975). Réflexions sur l'analyse de l'espace en géographie humaine: Colloque sur les fondements théoriques de la recherche en géographie. Torino: G, Giapichelli.

Raffestin, C. (1980). Pour une géographie du pouvoir. Paris: Litec.

Ramos, Ó. (1990). A la conquista del azúcar. Cali: Talleres gráficos de Impresora Feriva Ltda.

Reynoso, C. (2010). Análisis y diseño de la ciudad compleja. Buenos Aires: Sb.

Saldanha, A. (2008). Heterotopia and structuralism. Environment and Planning, 40.

Sánchez, G. (2008). Trujillo. Una tragedia que no cesa. Primer informe de memoria histórica de la Comisión Nacional de Reparación y Reconciliación, Bogotá: Planeta.

Sánchez, Ricardo. (2008). Las iras del azúcar: la huelga de 1976 en el Ingenio Riopaila. Historia crítica, 35. 
Sassen, S. (2007). Una sociología de la globalización. Buenos Aires: Katz editores.

Tapia, A. (2009). Algunos geosímbolos de Baja California. Identidad y memoria colectiva de la ruralidad. Culturales, 5(10).

Topinka, R. (2010). Foucault, Borges, Heterotopia: Producing Knowledge in Other Spaces, Foucault Studies, 9.

Urdinola, Z. (2013). Trabajo de campo, entrevistas, junio 2 [versión disponible en filmación: duración 4 horas].

Urdinola, Z. (2014). Trabajo de campo, entrevistas, junio 29 [versión disponible en filmación: duración 2 horas].

Van Dijk, T. (1996). Estructuras y funciones del discurso: Una introducción interdisciplinaria a la lingüística del texto y a los estudios del discurso. Décima edición. México: Siglo Veintiuno Editores.

West-Pavlov, R. (2009). Space in Theory, Kristeva, Foucault, Deleuze. Amsterdam, New York: Rodopi. 